\title{
Food system concepts and definitions for science and political action
}

\author{
For fruitful deliberations and concerted action at the science-politics interface, the concepts of food systems and \\ drivers of change need to be clearly understood and employed by all.
}

Joachim von Braun, Kaosar Afsana, Louise Ottilie Fresco, Mohamed Hassan and Maximo Torero

F ood systems exist at different scales: global, regional, national and local. Local food systems around the world are very diverse and location specific. They share some key features, but any attempt to change them should reflect their uniqueness resulting from traditions, cultures, economic structures and ecologies of locations. Change in food systems comes about through external and internal drivers, as well as through feedback mechanisms between these drivers. External drivers are, for instance, from climate or health systems, while internal drivers are, for instance, from productivity gains as a consequence of innovations or from changes in consumer behaviour.

The way in which changes in food systems impact sustainability in its diverse social, economic and ecological dimensions is critical. With the Sustainable Development Goals (SDGs) ${ }^{1}$ there is an accelerating momentum worldwide to adopt systems approaches to bring consumption and production patterns together to achieve sustainable development through an integrated approach to food systems.

\section{Defining and conceptualizing}

A practical definition of food systems should meet two essential criteria: (1) it should be suitable for the purpose at hand, which is to support global and national collective efforts to bring about positive change in food systems by accelerating progress on meeting the 2030 Agenda and the SDGs, in particular, to end hunger, improve diets and protect ecologies; and (2) it should be sufficiently precise to define the domains for policy and programmatic priorities but sufficiently general to not exclude any aspects of the economic, social and ecological dimensions of sustainability.

The significance of criterion 1 is that the definition should guide not only scientific inquiry but actions of all types toward a common purpose, namely, food systems change and, in the long run, even food systems transformation ${ }^{2}$. The point of criterion 2 is to avoid the intellectual



Fig. 1 | Interlinked systems. The food system in a positive systems concept with health, environmental, economic and innovation systems ${ }^{7,8}$.

hubris that accompanies many efforts of characterizing and graphically depicting food systems' complexities in great detail. Efforts to map food systems visually may help scientists as well as decision makers to identify key interactions and the mechanisms, both natural and social, that regulate those interactions. Yet, food systems maps that try to be fully comprehensive tend to collapse under the density and complexity of the interactions being described and analysed. At the other extreme, food systems maps and models that focus too narrowly on a reduced set of phenomena gain apparent explanatory power at the cost of realism, adequacy or the exclusion of important economic, social or biophysical environmental forces. There is no clearly defined pathway out of this dilemma. Much depends on the relevant policy question as well as on the context and scale of the food systems under consideration.

Food systems embrace the entire range of actors and their interlinked value-adding activities involved in the production, aggregation, processing, distribution, consumption and disposal (loss or waste) of food products originating from agriculture (including livestock), forestry, fisheries and food industries, along with the broader economic, societal and physical environments in which these activities are embedded $^{3}$. The range of actors includes, importantly, those from science, technology, data and innovation sectors ${ }^{4}$.

Sustainable food systems are those that contribute to food security and nutrition for all in such a way that the economic, social, cultural and ecological bases that generate food security and nutrition for future generations are safeguarded ${ }^{5}$. It should be noted that desirable food systems are necessary but not sufficient to assure good nutrition - even the best food system cannot assure good nutrition in a situation of poor hygiene, unclean drinking water, poor childcare and widespread infectious diseases. Moreover, the availability of plentiful and healthy food does not guarantee adequate consumption patterns or prevent excess body weight.

The concept of food systems transformation has been linked to the aspirations of the 2030 Agenda and refers to the objective of pursuing fundamental change in food systems, for instance, to aim 


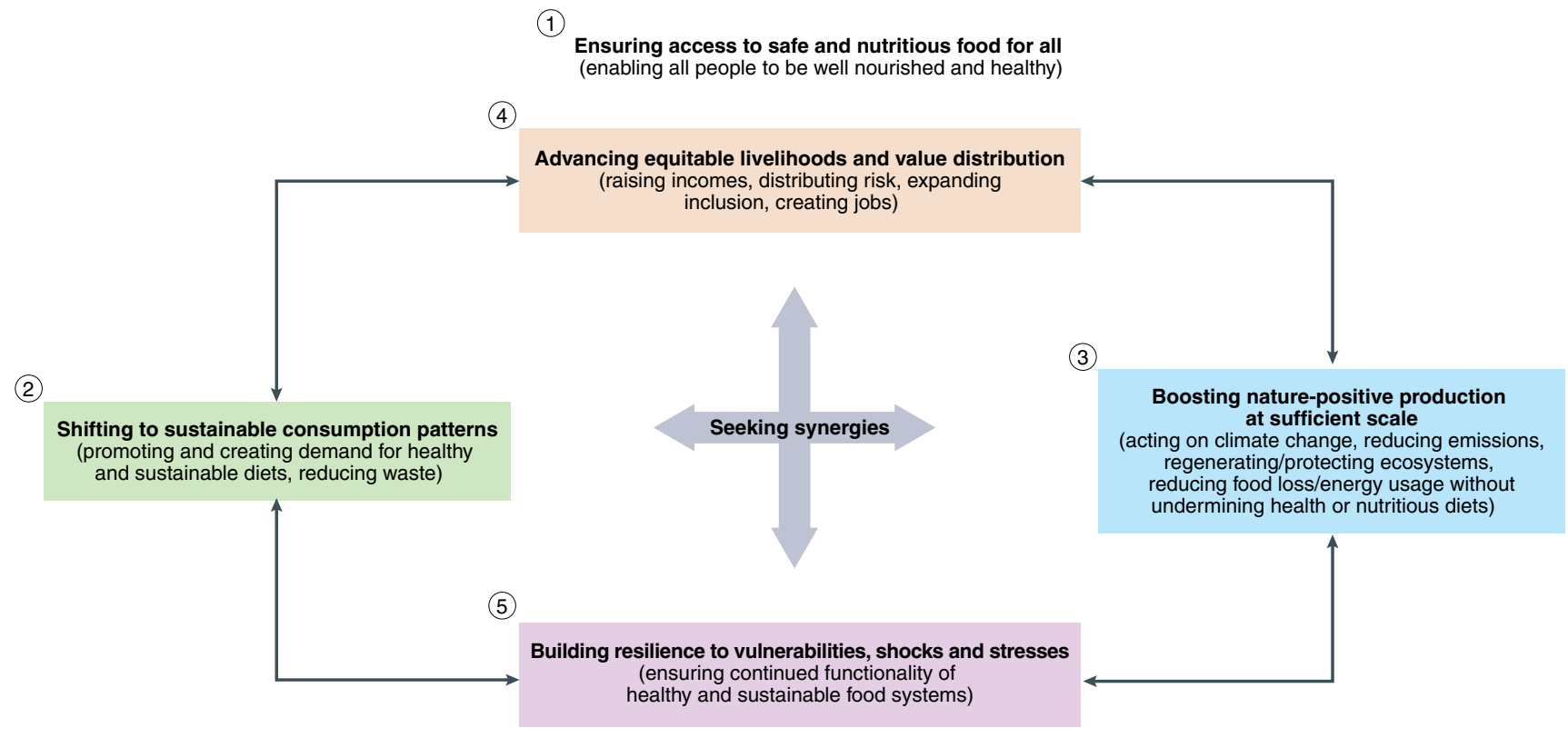

Fig. 2 | Food Systems Summit Action Tracks. The five Action Tracks are aligned in a normative perspective to the food systems concept.

for climate neutrality and achieving the SDGs. Transformation is a never-ending process in food systems, transition is the movement from one state to another, and evolution is the process of change. These are not interchangeable terminologies. Most food systems need all three.

Conceptualizing food systems entails defining system boundaries and system building blocks and linkages between them and understanding connections with neighbouring systems such as health, ecology, economy and governance, as well as the science and innovation systems (Fig. 1). Food systems are in a continuous state of change and adaptation. For the Food Systems Summit, this requires the identification of actions that enhance the positive side-effects or remediate or mitigate the negative side-effects of policies. The elimination of the net-negative externalities of food systems in terms of ecology and health costs would reveal the true costs and price of food. The concept of a sustainable circular bioeconomy as an overarching systems frame, in which food systems are embedded, could be considered in the solution-finding process.

\section{An action-oriented concept}

Systems can be conceptualized from a positive or from a normative perspective. The positive concept attempts to design systems structures and functions as they occur in the current real world and identify where desirable systems change can be introduced. The normative concept postulates a set of objectives and aims to shape the systems to serve the stated objectives. Both concepts aggregate and simplify real-world structures and processes. Neither of these approaches escapes the yardstick of scientific evidence. For theoretical clarity of the underlying value judgments, however, the two approaches need to be distinguished. As the Food Systems Summit is based on clearly stated objectives already defined in the SDGs, a normative approach is justified. Yet, normative approaches need to be put to the test by positive approaches to avoid steering into a dead end of unrealistic wishful thinking. Thus, normative and positive approaches are complementary. To build upon existing efforts, we suggest a concept of food systems that may help to frame action-oriented agenda setting, such as the one reflected in the five Action Tracks for the Food Systems Summit in support of the SDGs. These Action Tracks are described as: (1) ensuring access to safe and nutritious food for all (enabling all people to be well nourished and healthy); (2) shifting to sustainable consumption patterns (promoting and creating demand for healthy and sustainable diets and reducing waste); (3) boosting nature-positive production at sufficient scale (acting on climate change, reducing emissions and increasing carbon capture, regenerating and protecting critical ecosystems, and reducing food loss and energy usage, without undermining health or nutritious diets); (4) advancing equitable livelihoods and value distribution (raising incomes, distributing risk, expanding inclusion and creating jobs); and (5) building resilience to vulnerabilities, shocks and stresses (ensuring the continued functionality of healthy and sustainable food systems).

The five Action Tracks capture key opportunities and challenges of food systems and relate to one or more food system components, but they do not define the concept of food systems as such. Therefore, pursuit of the Action Tracks needs to take into account an overarching food systems concept. Pursuing each Action Track in isolation from the others would lead to inefficient solution proposals that neglect system-wide effects. We thus offer a perspective that attempts to position the five Action Tracks in a food systems framework (Fig. 2): we expect food security and nutrition, livelihood improvements, and production that is environmentally sustainable; we want resilience to shocks (that is, low variability, and a quick recovery from negative shocks); and we know that consumption patterns are a powerful lever for change. Ensuring access to safe and nutritious food for all (enabling all people to be well nourished and healthy) is supported by the other four Action Tracks, yet improved nutrition also feeds back into the other four Action Tracks. The Action Tracks need to consider functional relationships among them in a systemic way.

The systems perspective must not overlook some key cross-cutting issues and themes that need due attention, for example, 
COVID-19 has highlighted the intertwining of food and health systems. Science and new and emerging technologies and innovations, including gene editing, digitization, the Internet of Things and artificial intelligence, are critical for improving the productivity, efficiency, equity and sustainability of food systems. The role of women and gender are important determinants for productive, healthy and sustainable food systems and are fundamental for equity. Trade, market structures and the dynamics of food industries require policy attention ${ }^{6}$. And there is a tendency to think of food systems as terrestrial systems only, but it will be vital to broaden the understanding of food systems to include their links to water cycles, oceans and fisheries.

\section{Concluding remarks}

The discourse on food systems must not abstract from the issue of culture and values, otherwise it would seem to be merely a technical question. This applies especially - but not only - to the greatly diverse indigenous food systems and the culture and knowledge embedded in them.

The Food Systems Summit needs to facilitate action to overcome system failures that contribute to hunger, malnutrition and obesity problems; to the ecological problems of deforestation, greenhouse gas emissions, biodiversity losses and species extinctions; to the problems of poor livelihoods in farming communities, especially for women and youth; and to the fundamental issues of food-system-related violations of rights the human right to food, broadly defined. The Summit needs to come up with visions for food system transformations in their respective contexts. While a strong sense of urgency is called for due to the malfunction of big food systems, the time horizon for the food system transformations needs to reach far beyond 2030, given demographic change, climate change, technological change and linkages between people and nature in the Anthropocene.

If food systems shall deliver on the stated objectives (the SDGs), the Food Systems Summit needs to be open to new thinking and concepts and to establishing new institutional and organizational arrangements. Addressing symptoms of system failures will not be sufficient. Investing in science is essential to innovate, develop and implement game-changing propositions that fit the respective food system contexts. Science and policy have a lot to gain from cooperating through a strong and effective science-policy interface to help guide the follow up to the Summit ${ }^{7}$.

Joachim von Braun (D) 1 , Kaosar Afsana², Louise Ottilie Fresco ${ }^{3}$, Mohamed Hassan ${ }^{4}$ and Maximo Torero ${ }^{5}$

${ }^{1}$ Center for Development Research, Bonn University, Bonn, Germany. ${ }^{2}$ BRAC James P Grant School of Public Health, BRAC University, Dhaka, Bangladesh. ${ }^{3}$ Wageningen University \& Research Executive
Board, Wageningen, the Netherlands. ${ }^{4}$ The World Academy of Sciences for the Advancement of Science in Developing Countries (TWAS), Khartoum, Sudan. ${ }^{5}$ Food and Agriculture Organization of the United Nations (FAO), Rome, Italy.

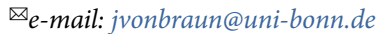

Published online: 15 September 2021 https://doi.org/10.1038/s43016-021-00361-2

References

1. United Nations Sustainable Development Goals (2020); https://go.nature.com/315xhJD

2. von Braun, J. et al. Ending Hunger by 2030 - Policy Actions and Costs (Center for Development Research, 2020); https://go.nature. com/3zXLxul

3. Nguyen, H. Sustainable Food Systems: Concept and Framework (FAO, 2018); https://go.nature.com/3hgKcHP

4. Herrero, M. et al. Nat. Food 1, 266-272 (2020).

5. Global Panel on Agriculture and Food Systems for Nutrition Foresight 2.0 (2020); https://go.nature.com/3hfHZMO

6. OECD Making Better Policies for Food Systems (2021); https://go.nature.com/3BUyMRH

7. InterAcademy Partnership Opportunities for Future Research and Innovation on Food and Nutrition Security and Agriculture. The InterAcademy Partnership's Global Perspective (2018); https://go.nature.com/3yWmrL0

8. von Braun, J. in Agriculture and Rural Development in a Globalizing World (eds Pingali, P. \& Feder, G.), Ch. 14, 273-291 (Routledge, 2017)

Acknowledgements

We gratefully acknowledge the peer review of a background paper on which this Comment is based by reviewers $\mathrm{P}$.

Pinstrup-Andersen (Copenhagen University), P. Scheelbeek (London School of Hygiene \& Tropical Medicine), M. Dev (Indira Gandhi Institute of Development Research), Z. Jing (Nanjing Agricultural University) and M. Toure (The World Academy of Sciences).

Competing interests

The authors declare no competing interests. 